\title{
El proceso de intervención en medio abierto: la libertad vigilada
}

\section{Pere Amorós}

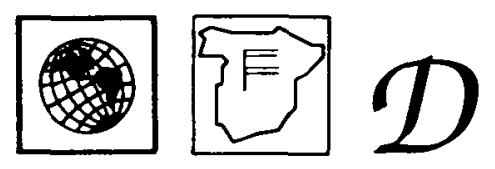

El autor describe una propuesta de intervención con menores en Medio Abierto, llevada a cabo en Cataluña, y centrada en la figura de los Delegados de Asistencia al Menor (DAM). En el articulo se indican las fases que caracterizan el proceso de intervención (fase inicial, fase intermedia y fase final), señalando en cada una de ellas los roles que el DAM asume, los diferentes tipos de estrategias y habilidades que utiliza, y los roles que el menor debería desarrollar.

La necesidad de ir adecuando unas medidas judiciales para los jóvenes infractores que estuvieran más de acuerdo con la realidad, ha facilitado que se fueran ampliando las medidas que pudieran aplicarse en el propio medio del joven. Éste hecho ha surgido de las recomendaciones de organismos internacionales como la Convención de Derechos del Niño de las Naciones Unidas, las Reglas de Beijing o las propia Recomendación (87)20 del Comité de Ministros del Consejo de Europa, que recomiendan medidas que pueden aplicarse en el propio entorno del menor, es decir en medio abierto, reservando el internamiento como último recurso.

En este sentido en Cataluña desde el año 1982 se crearon los Delegados de Asistencia al Menor (DAM) para poder desarrollar programas en medio abierto y en particular los de Libertad Vigilada. La Libertad Vigilada consiste en la intervención socio-pedagógica caracterizada por una combinación de asistencia educativa y control, que se realiza en el propio entorno del menor utilizando todos los recursos de que disponga la comunidad.

La necesidad de ir estructurando la mejor forma de llevar a cabo la intervención, o sea la metodología de intervención, fue lo que motivó que un grupo de profesores universitarios y todos los Delegados de Asistencia al Menor llevaran a cabo una investigación cooperativa a lo largo de dos años (Amorós, 1993). A partir de esta investigación surgieron los diferentes aspectos que conforman la metodología de intervención en Medio Abierto (objetivos, proceso de intervención, instrumentos de evaluación). En este artículo presentamos una parte de esta investigación referida al Proceso.

La determinación de las características del proceso de intervención en Medio Abierto ha representado uno de los aspectos más laboriosos Esta dificultad se ha centrado en que las diferentes estrategias, técnicas y habilidades que utilizan los DAM 
provienen de las aportaciones realizadas desde diferentes corrientes teóricas del ámbito psicológico, pedagógico y social. Por lo tanto la estructura de este proceso de intervención hay que verla desde un planteamiento ecléctico, abierto y flexible. Ecléctico porque utiliza aportaciones de corrientes científicas diferentes. Abierto porque sólo presenta un marco de referencia general, un punto de partida, una orientación para la intervención. Flexible porque contempla la posibilidad de adaptaciones a las necesidades individuales que puedan presentar los menores.

\section{FASES DEL PROCESO DE INTERVENCION}

\section{Fase Inicial}

Lo que se pretende en la fase inicial es conocer y motivar al menor, la familia y el entorno para que colaboren en la elaboración e implantación del Proyecto Educativo individualizado.

\section{Roles del Dam}

Los roles del Dam en esta primera fase son los siguientes:

- Motivación del menor, la familia y el entorno.

- Información de la medida judicial.

- Conocimiento del menor, la familia y el entorno (Evaluación inicial).

- Elaboración del PEI y el informe al juez.

- Implantación del plan de trabajo.

- Fomentar el nivel de conocimiento.

Motivación del menor, la familia y el entorno

La intervención en Medio Abierto, no sólo concibe la intervención sobre el menor, sino también sobre su familia y su entorno. La posible colaboración de la familia y del entorno en el plan de acción facilitará enormenente los progresos del menor. Por ello en esta fase es muy importante detectar, en primer lugar, el nivel de implicación, colaboración y aceptación que presenta la familia y el entorno y a partir de ello crear los elementos necesarios para motivarlos a participar y colaborar en los planes de trabajo que se realicen para el menor.

La motivación configura un elemento básico en el inicio de un proceso de intervención. El Delegado intenta motivar a través de : la atención, el interés, el deseo y la acción. Lo primero que realiza es llamar la atención tanto del menor como de su familia, les prepara para que puedan recibir una información. Para ello el Delegado utiliza diferentes procedimientos (cartas, llamadas telefónicas, contactos personales, etc.). Una vez que ha podido captar la atención se procede a informar de las características de la intervención explicándole entre otros temas las ventajas y la ayuda que pueden recibir con esta intervención. De esta información y argumentación es de donde puede surgir el interés por parte del menor y la familia. Después que el Delegado ha informado y conseguido interesar a las personas sobre su proyecto irá aumentando sus niveles de relación y de información para que surja en el menor, su familia y el entorno un deseo sobre el tema. A partir de este deseo, se puede ir desarrollando todo el proceso de intervención.

Conocimiento del menor, la familia y el entorno (Evaluación inicial)

El proceso de intervención del Dam, para ejecutar una medida de Libertad Vigilada, puede realizarse después de una fase de Observación en Medio Abierto o iniciar el proceso sin que haya existido esta fase previa. En el caso de que se hubiera realizado esta fase previa de Observación, el Dam ya partiría de un conocimiento del 
menor, la familia y el entorno, en caso contrario, tendría que llevar a cabo esta función.

La evaluación inicial que realizan los DAM corresponde a la comprensión que nos hacemos de la realidad que se percibe a partir de los diferentes puntos de referencia como son: el menor, la familia y el entorno. La comprensión de un fenómeno aparece como una operación progresiva y continua. De esta forma la evaluación es un proceso por el cual llegamos a comprender al sujeto, su universo y la significación que revisten para él sus interacciones con este universo. La evaluación es un concepto dinámico que permite no sólo saber, sino igualmente preveer y tomar decisiones.

Elaboración del Proyecto Educativo Individualizado (PEI) y el informe al Juez

En la elaboración de un Proyecto Educativo Individualizado se recogen las características del menor, la familia y el entorno, junto con una valoración y propuesta de plan de acción. Este proyecto es la planificación prevista para llevar a cabo la intervención sobre los diferentes ámbitos de intervención del DAM. Es un proyecto abierto, flexible y en constante revisión de acuerdo con las diferentes evaluaciones que se irán realizando a lo largo del mismo.

El Proyecto Educativo individualizado no sólo refleja el plan de trabajo que el DAM puede desarrollar con aquel menor, sino que también incluye las actuaciones sobre los otros ámbitos de intervención y la posible colaboración, en este proyecto, de los profesionales y de los recursos sociales existentes en aquella zona. Se intenta que el menor colabore en la elaboración de todo el proyecto, de esta forma los compromisos pueden ser mejor asumidos.

El DAM al ejecutar una medida por mandato judicial tiene que facilitar periódicamente al juez informes sobre las características de la evolución de la intervención. A lo largo del proceso se realizan tres tipos de informes: el inicial, el de seguimiento y el final.

Implantación del Proyecto Educativo Individualizado (PEI)

La puesta en práctica del PEI es un reto para el Delegado y para el menor ya que indica si las previsiones realizadas se adaptan a las características del menor y de su entorno. Pretende la consecución de los objetivos, la adecuación de las estrategias y técnicas y los controles necesarios para ir readaptando el proceso.

\section{Fomentar el conocimiento de la realidad}

Es una tarea del DAM dirigir adecuadamente la toma de conciencia de forma que permita al menor comprender mejor su problema y su realidad actual y pueda verlo desde objetivos de cambio específicos que producirán una mejoría.

A menudo el DAM inicialmente hace algo más que atender y clarificar las percepciones de lo que está experimentando el menor. Busca también respuestas para los interrogantes ¿Qué está ocurriendo exactamente en la vida cotidiana de este menor? ¿ De qué modo podrían responder otras personas a los sentimientos y pensamientos del menor?. Es decir, la atención se dirige a esta persona y a los detalles particulares del ambiente en que vive.

\section{Roles del menor}

La colaboración en el proyecto y el conocimiento de su realidad son dos de los roles que el menor tendría que desarrollar en esta fase. Roles que irán consolidando y aumentando en fases posteriores. Cuanto mayor nivel de colaboración y de conocimiento de su realidad se consiga por parte del menor, mayores serán las posibilidades para alcanzar todos aquellos objetivos específicos que hayamos diseñado en su PEI. Según Spivack, Platt y Shure (1976), McGuire y Priestley (1985) el que las personas 
adquieran un conocimiento de su realidad es muy importante para que tengan conciencia de que los problemas existen y que tienen capacidad para solucionarlos.

La colaboración

Que el menor alcance un buen nivel de colaboración es un objetivo que facilitará todo el proceso. La colaboración del menor puede plasmarse en varios aspectos:

- Intercambio de información para así facilitar al Delegado un mejor conocimiento sobre sus necesidades, intereses, dificultades y potencialidades.

- Elaboración de su proyecto educativo. Dicho proyecto elaborado con la colaboración del menor y que con la ayuda del DAM ha de ser factible.

- Implantación del plan de trabajo. Es decir, respetar los compromisos que implica la puesta a punto del plan de trabajo.

El conocimiento de su realidad

Un elemento importante para que una persona pueda experimentar un cambio, es que tome conciencia de cuál es su situación y cuáles son aquellas circunstancias que le han producido la situación conflictiva o problemática. El conocimiento de su realidad es un proceso que permite al menor llegar a tener una comprensión de su situación y conocer en dónde está. Esta exploración facilitará la comprensión más profunda y, sobre todo, la toma de decisiones para resolver sus conflictos y desarrollar sus potencialidades. El DAM a través de la utilización de una serie de estrategias y habilidades intentará ayudar al menor a lograr este conocimiento.

\section{Tipos de estrategias, técnicas y babilidades}

Para la realización de sus roles en esta primera fase, el DAM debe utilizar diferentes estrategias y poner en juego determinadas técnicas y habilidades. Las estrategias que utiliza el DAM en esta fase van dirigidas fundamentalmente a conseguir un conocimiento y una motivación del menor, la familia y el entorno; a elaborar el plan de acción y a facilitar una mejor relación e integración del menor con la familia y el entorno (tanto con sus iguales como con los recursos existentes).

Estrategias, técnicas y habilidades de relación directa

La entrevista es por excelencia la técnica más utilizada por el DAM. Usan esta entrevista con dos fines: recoger información y establecer una relación de ayuda. La entrevista es una fuente importante de información para la evaluación. Esta informàción es útil para captar lo que subyace bajo los aspectos de la actividad del menor, genera descripciones detalladas y brinda una comprensión holística de los puntos de vista del menor.

A través de la entrevista el delegado intenta que se consigan la gran mayoría de sus objetivos previstos. A lo largo de las entrevistas el DAM utiliza habilidades que le facilitarán la consecución de los objetivos. Entre las más significativas en esta fase destacaríamos:

Captación. Un aspecto básico en el inicio de una relación es conseguir captar la atención de las personas que tienen que estar implicadas en el proceso. Si se consigue captar la atención estamos creando un marco adecuado para ofrecerle información y de esta forma desarrollar el interés y el deseo para continuar el proceso de intervención. Lo primero que realiza el DAM es llamar la atención tanto del menor como de su familia. Les prepara para que puedan recibir una información. Podríamos decir que la intervención del DAM se inicia a partir de un mandato judicial que recibe a través de su Jefe de Zona, el cual le asigna el caso. Anteriormente a esta situación el Juez ha notificado la medida al menor y al Servicio de Medio Abierto. El Servicio también notifica por escrito al menor la medida y le indica que próximamente un DAM se pondrá en contacto con él. El DAM a través de una carta, de una llamada 
telefónica, o de unos contactos personales intenta captar la atención del menor y su familia.

Una vez que se ha podido captar la atención, se procede a informar de las características de la intervención: Ventajas que les puede aportar, disponibilidad del DAM, deberes y derechos de las partes, etc. De esta información y argumentación es de donde puede ir apareciendo el interés por parte del menor y la familia.

La escucha. El objetivo de la escucha, es prestar una atención plena al menor centrando toda nuestra atención en todo aquello que trata de expresar verbalmente. Ello puede permitir al menor sentirse que alguien le escucha y se interesa por él. El DAM utiliza la escucha para ofrecer una posibilidad de expresión y comprensión. Esto facilita un clima de confianza y seguridad tan necesario para iniciar cualquier proceso de intervención.

La observación. A través de la observación, el DAM puede comprender mejor el contexto donde tiene lugar las actividades del menor desde una perspectiva más holística. El objetivo de la observación es ver y entender las conductas del menor y la familia en diferentes contextos. La observación que utiliza el DAM suele ser de tipo participante ya que incide sobre el medio, y éste incide sobre el DAM, lo que obliga a conjugar la implicación personal con un distanciamiento adecuado de la realidad a observar.

La empatía. La empatía es otra de las habilidades más usadas por los DAM. A través de la empatía el Dam es capaz de objetivizar y comprender lo que vive el menor y comunicándole esta comprensión de la forma más adecuada, de acuerdo con el momento presente. La empatía ayudará al menor a conocer de su realidad, sus experiencias y sentimientos. Comprenderá los modos de vida y de relacionarse que hayan sido favorecedores o los que son inefectivos o inadecuados y podrá comunicar al mismo tiempo lo que esta situación representa para él.

En esta fase la empatía se puede utilizar de una forma en que las respuestas que facilite el DAM sean esencialmente intercambiables con las del menor, en cuanto que ellas expresan esencialmente los mismos sentimientos y significados. En esta fase no se intentará ofrecer respuestas que añadan un significado y un sentimiento más profundo.

La confianza. Una de las habilidades que más citan los delegados en esta primera fase es el de ofrecerle confianza al menor para ganar su confianza. Esta habilidad consiste en ayudar al sujeto a evolucionar personalmente en el sentido de una mayor capacidad de resolución de problemas de su existencia. Esta evolución, creemos nosotros, se puede realizar en la medida en donde el DAM en la relación de ayuda acepta al sujeto tal cual es y le manifiesta una confianza realista en sus capacidades de adaptación .

La aceptación. Implica el aprecio de la dignidad y valores del menor. En esta fase es importate la utilización de una aceptación que permita al menor expresarse con toda libertad. El DAM facilita que la persona se pueda expresar tal cual es, sin realizar juicios de valor o actitudes censurables que pudieran tener un efecto restrictivo en las expresiones del menor. El DAM debe transmitir una consideración hacia la posición del menor y un interés hacia sus sentimientos, experiencias y potencialidades.

La aceptación no supone una aprobación de las conductas. El respeto o aceptación no se puede situar dentro de una valoración de bueno o malo, negativo o positivo. Lo importante es la aceptación del otro tal como es y no tal como quisieramos que fuera, y la creencia en su capacidad para resolver problemas.

La concreción. Es preciso ayudar al menor a que pueda concretar y especificar sus dificultades o conflictos. Una de las características que manifiestan los DAM es que a los menores les cuesta centrarse, que generalizan mucho sus sentimientos y emociones y que en ocasiones las expresiones o verbalizaciones no están relacionadas con sus sentimientos y experiencias. Es por ello que en esta fase el Dam utiliza la concreción para ayudar al menor a que no se esconda en las generalizaciones y pueda 
discutir de una forma abierta y concreta sus sentimientos y experiencias. El DAM ayuda con sus respuestas al menor a expresar el núcleo específico de su problemática, sin encubrirlo con hechos o sentimientos más o menos importantes.

Los refuerzos. Los refuerzos, son otras de las técnicas utilizadas por los DAM. Los refuerzos se utilizan para aumentar la frecuencia de la conducta deseable pero infrecuente. Típicamente el refuerzo se ha definido como cualquier evento que sirve para incrementar la probabilidad de una conducta.

Por lo general los DAM utilizan diferentes tipos de refuerzos: Refuerzos materiales, sociales y autorefuerzos. El refuerzo material, como cosas, actividades o dinero; el refuerzo social, como alabanza o aprobación y el auto-refuerzo, que supone la autoevaluación positiva por haber realizado algo.

Estrategias de relación indirecta: La utilización de los recursos

La Intervención en Medio Abierto se define como la atención individualizada a un menor, encaminada a la integración de éste en la sociedad, incidiendo en la familia y utilizando los recursos del entorno social, con estrecha coordinación con las diferentes instituciones, entidades y profesionales de la zona.

La utilización de los recursos normalizados o especializados forma parte de las estrategias más utilizadas por los DAM. La temporalización de las medidas ha supuesto un mayor uso, por una parte, para intensificar la intervención sobre el menor y, por otra, para tener integrado este menor en un recurso que le permita una continuidad una vez finalizada la medida judicial.

Esta derivación comporta: un conocimiento amplio por parte del DAM de los recursos existentes en la zona y de las características de los mismos, una coordinación con todos aquellos profesionales que colaboran en el caso, un apoyo al menor y una evaluación de todo el proceso.

\section{Fase Intermedia}

En esta fase lo importante es continuar con el plan de trabajo, asegurarnos de que el menor aprende y puede utilizar sus habilidades para tratar sus problemas. Ver que utiliza sus comportamientos en todas las áreas de su vida.

\section{Roles del Dam}

- - Aumentar el nivel de comprensión y conseguir la estabilidad del proceso de intervención.

- Establecer los periodos o momentos de la evaluación formativa.

- Reforzar el nivel de colaboración del menor, la familia y el entorno.

Aumentar el nivel de comprensión y conseguir la estabilidad del proceso de intervención

Si realmente se ha iniciado el proceso de una forma adecuada y no han surgido variables que dificulten la evolución del menor el DAM intenta buscar la estabilidad del proceso. Estabilidad referida a consolidación y evolución de los objetivos y a una mayor comprensión de la situación. Es importante que el menor personalice sus problemas y personalice sus sentimientos. La estabilidad en el proceso de intervención se relaciona tanto con la intervención sobre el menor, como con el mantenimiento de los contactos y relaciones necesarias con la familia y los recursos del entorno.

\section{Establecer los periodos o momentos de la evaluación formativa}

La evaluación, al igual que el proceso de intervención, es un proceso abierto, flexible y dinámico. Los Delegados manifiestan que las propuestas de intervención 
tienen que adaptarse continuamente a las diferentes variables que entran en juego en la dinámica del menor. Lógicamente estos cambios tienen que ser producto de una evaluación del proceso. Esta evaluación nos permitirá determinar la implementación del plan de trabajo y cuáles son aquellos cambios más oportunos que se tengan que realizar. Cambios que tanto pueden ir dirigidos a los objetivos, como a las estrategias o técnicas de intervención.

Reforzar el nivel de participación del menor y la familia

$\mathrm{La}$ intervención sobre el menor es el elemento clave del DAM. Se concibe que la propia intervención tendrá previsto al menor y su contexto. La participación de los miembros de la familia y de otros profesionales, apoyando y reforzando la labor, es un elemento importantísimo para facilitar su evolución. El DAM en la medida que sea posible intenta que exista, cada vez más, un mayor nivel de participación y colaboración.

\section{Rol del menor}

Responsabilidad

«La responsabilidad individual, en el marco de la Justicia juvenil, se aplica en el sentido de situar al sujeto infractor ante sus propios actos, señalando las consecuencias que éstos tienen para la víctima y para la sociedad. Pedir esta responsabilidad significa reconocer la pertenencia de sus acciones y respuestas, y señalar el sentido, valor y consecuencias que estas acciones tienen sobre los otros y sobre ellos mismos» (Proyecto Técnico de Medio Abierto,1991, p 14).

En esta fase, se desea que el menor alcance una mayor responsabilidad en sus actos y en su plan de trabajo. La responsabilidad en todo el plan de intervención le puede servir de ayuda para alcanzar de una forma efectiva los objetivos previstos y prepararle para la fase final, en que el menor tendrá que terminar su relación y dependencia con el DAM. Junto con este objetivo propio de la fase intermedia se irán trabajando todos aquellos objetivos específicos que se hayan diseñado en el Proyecto Educativo Individualizado.

\section{Tipos de estrategias, técnicas y babilidades}

En cuanto a las estrategias, son parecidas a la etapa anterior. Se consolidan aquellas que mejor ayudan al menor y se establecen los contactos adecuados para realizar un seguimiento y una motivación de todas las personas implicadas en el proceso. Tal vez uno de los elementos más significativos que pueden darse en esta etapa y en la fase final son la participación del menor en actividades educativas que puedan realizarse dentro o fuera de su entorno natural. Estas actividades permiten ampliar el marco de relación del menor y confrontar sus comportamientos en otros entornos.

Estrategias, técnicas y habilidades de relación directa

En cuanto a las técnicas se sigue manteniendo la entrevista como elemento básico de la relación. Una entrevista que se irá utilizando tanto desde una perspectiva informal como desde una perspectiva dirigida.

La autocrítica. Dentro de un modelo de responsabilidad, es importante utilizar esta habilidad para que el menor reflexione sobre su situación personal y vaya sentando las bases para la futura toma de decisión- libre y responsable- acerca de la voluntad o no voluntad de cambio.

La observación y la escucha. Son habilidades que se utilizan en cada una de las fases. En esta fase intermedia la escucha vuelve a coger un gran protagonismo ya que a través de ella podernos $i_{i}$ consolidando un buen nivel de confianza. La confianza es 
un sentimiento que se va facilitando a través de la escucha, ya que la persona que se siente escuchada, se siente aceptada como persona. Tiene una sensación de placer y ésto le facilita tener más claros sus pensamientos, sentimientos o conflictos.

La empatía. Con una comprensión profunda por parte del DAM, el menor puede entenderse mucho mejor a sí mismo. Es importante en esta fase que el DAM intente centrar toda su atención en la exactitud de la percepción de los sentimientos del menor y sobre todo que le comunique de una forma adecuada esta percepción. El modo en que podamos hacer llegar el mensaje al menor facilitará enormemente su nivel de comprensión y ayudará a alcanzar unos niveles superiores de confianza con el DAM. En esta fase el DAM facilitará todas unas respuestas empáticas que añadan considerablemente un significado y sentimiento más profundo.

La confianza. Si en la fase anterior, la confianza era utilizada como una de las habilidades importantes, en esta fase recobra todo el sentido. La confianza a lo largo del proceso de relación de ayuda se manifiesta en el DAM por una actitud :

- de confianza consigo mismo, con sus conocimientos profesionales, con su experiencia personal y con los recursos que tiene a su disposición. Si la evolución del menor ha sido positiva, esta confianza en sí mismo la ha ido adquiriendo y reforzando.

- de confianza con el menor, que aunque sea una persona que presenta algunas dificultades, dispone de unas fuerzas o potencialidades que con nuestra ayuda le pueden permitir encauzar sus problemas. Del mismo modo un mayor conocimiento de las características del menor puede facilitar una mayor confianza en sus actuaciones. Estos niveles de confianza se transmitirán al menor para que vaya adquiriendo una mayor confianza consigo mismo y con el DAM.

La aceptación. La relación de ayuda bajo el aspecto de la aceptación o la consideración, es el resultado de interacciones apropiadas conscientes de respeto mutuo entre el DAM y el menor. Respeto vivido, cada uno a su nivel, por ellos mismos, por el otro y por su propia relación que se manifiesta diferente en función de la evolución de esta consciencia, la cual fundamenta su respeto para otras relaciones.

En esta fase el DAM utiliza un respeto más condicionado, es decir, que busca respetar aquellas actitudes o comportamientos que pueden facilitar un cambio al menor.

La confrontación. La utilización de la habilidad de la confrontación, tal vez, es una de las habilidades que pueden representar un mayor riesgo si no se utilizan de una forma adecuada y en el momento oportuno. Se sugiere que la confrontación no se tiene que urilizar en unas primeras fases ya que puede facilmente romper la relación al no estar preparado el menor para recibir esta confrontación de su situación. Es por ello que el DAM utiliza la confrontación en esta fase, en la cual situa al menor delante de una realidad que no ha sido considerada hasta el momento, y ello puede tener un efecto para él. La confrontación surge cuando el educador pone al sujeto de cara a las realidades que pertenecen a su campo de conciencia y que han sido evocadas implicitamente por el sujeto, pero sin haber sido miradas de cara por éste.

Técnicas conductuales y cognitivas

En el proceso de intervención los DAM no utilizan técnicas que provengan de una sola ciencia o teoría científica sino que adaptan para sus fines técnicas de corrientes diferentes. Al igual que en la primera fase la técnica de los refuerzos se sigue utilizando.

En cuanto a las técnicas cognitivas, hemos podido constatar que últimamente se han empezado a utilizar por parte de algunos DAM (Guasch, 1991). Resulta el más reciente desarrollo dentro de la modificación de conducta, en cuyos modelos se enfatizan la importancia de los procesos cognitivos y de los sucesos privados como mediadores del cambio conductual: los presupuestos implícitos, las interpretaciones y atribuciones de la propia conducta, autovaloraciones, metacogniciones y procesos 
similares son considerados como los determinantes de los trastornos conductuales y del cambio conductual.

Con estas técnicas se intenta realizar un cambio conductual, pero que debe afectar a diversos niveles «cambio de forma de comportase» que implica la supresión de conductas desadaptativas y su sustitución por conductas adaptativas; « cambio en la forma de pensar", por el que se sustituyen formas de pensamiento inadecuadas y desadaptativas por formas de pensamiento más realisticamente orientadas y adaptativas, y, asimismo, un control adecuado de sus habilidades (incremento) y de las condiciones medio ambientales en las que se desarrolla su vida.

Los desafíos. Los desafíos son utilizados para "provocar" al menor a realizar una actividad o una conducta determinada. En ocasiones y ante la falta de iniciativa de los menores o la débil predisposición para realizar una actividad, el DAM puede utilizar el desafío en el sentido de provocar al menor un efecto de participación. Por ejemplo, insinuarle que él no será capaz de realizar una actividad determinada.

Estrategias de relación indirecta: La participación en actividades educativas

La participación del menor en actividades educativas forma parte de las estrategias utilizadas por una gran parte de los Delegados, si bien, existen diferentes posicionamientos entre los equipos de delegados en cuanto a que las actividades estén organizadas por la comunidad o por el propio equipo, y a la implicación directa o no del Delegado.

Entre las diversas actividades propias o ajenas que han participado los menores podemos citar a modo de ejemplo: excursiones de uno o varios días, visitas a centros de interés cultural, deportivo o artístico, asistencia a películas, actividades de verano (natación, salidas en crucero, colonias), actividades de invierno (montañismo, esquí,..), viajes de intercambio con menores de otras nacionalidades, etc.

Como conclusión en relación con las actividades educativas podemos afirmar, como indican un grupo de delegados, que «el valor y el alcance de las actividades educativas no reside en ellas mismas sino principalmente en su articulación en un proceso educativo continuado...

\section{Fase Final}

La fase final se caracteriza por la preparación del menor a una mayor autonomía e integración social y a preparar la despedida. EI DAM, a lo largo de esta fase, pretende fundamentalmente captar la vivencia del menor ante esta separación, ver la capacidad de autonomía que puede haber ido adquiriendo y hasta qué punto el menor puede utilizar los recursos de la sociedad para que complementen o continuen la labor iniciada.

\section{Roles del Dam}

- Preparar la despedida.

- Información de la finalización.

- Captar la vivencia del menor ante la finalización.

- Hacer conocer las diferencias de las respuestas judiciales, según sean de a jurisdicción de menores o ordinaria. zados.

- Prever la continuidad o derivación a los recursos normalizados o especiali-

— Evaluación sumativa o de resultados.

\section{Preparar la despedida}

Anteriormente era el DAM el que, después de un largo proceso de intervención, determinaba, a partir de toda una serie de criterios, proponer al juez la finaliza- 


\section{4}

ción de la medida. Actualmente todas las medidas de Libertad Vigilada están temporalizadas. Ello permite al DAM preveer con exactitud la finalización de esta medida. Este conocimiento permite una mejor preparación de la despedida, en donde se puede hacer una reflexión sobre el trabajo realizado y las perspectivas de futuro de cara al menor.

Varios son los aspectos que abordan los DAM para preparar esta despedida:

\section{La información de la finalización}

El DAM informa y recuerda al menor la fecha de finalización de la medida, ya que se ha podido constatar que muchos menores van perdiendo la noción del tiempo de duración de la misma.

\section{Captar la vivencia del menor ante la finalización}

Los DAM señalan que es muy importante poder captar la vivencia del menor ante la finalización, ya que a partir de ella se pueden preveer la posibilidad o no de continuar en otro tipo de recursos o en los casos que se crea oportuno continuar la intervención a través de la denominada «postmedida». Ésta consistiría en mantener un nivel de relación con el DAM pero bajo una situación de voluntariedad.

Hacer conocer las diferencias de las respuestas judiciales, según sean de la jurisdicción de menores o ordinaria

Otro de los aspectos que señalan los DAM, en relación con la preparación de la despedida, es el de informar lo más claro posible sobre las diferencias de las respuestas judiciales dirigidas a los adultos y las dirigidas a los menores.

\section{Prever la continuidad o derivación a los recursos normalizados o especializados}

La utilización de los servicios normalizados o especializados es un hecho muy frecuente por parte de los menores en Libertad Vigilada. La posibilidad que puedan continuar en estos servicios u otros servicios una vez acabada la medida, representa, por lo general, uno de los objetivos previstos en el plan de trabajo inicial. La realidad de la utilización de estos recursos está condicionada lógicamente por la existencia o no de los mismos y por la posibilidad de que los servicios puedan o quieran aceptar a los menores y ofrecerles una atención adecuada.

\section{Evaluación sumativa o de resultados}

La evaluación es un proceso dinámico y continuo que se ha ido realizado a lo largo de toda la intervención. Al finalizar la misma, se realiza una evaluación sumativa o de resultados. El DAM intenta evaluar, a partir de las informaciones recogidas, la evolución de los sujetos (ya sea menor o familia) y su propio proceso de intervención, con sus objetivos, estrategias y técnicas. En esta evaluación se tiene en cuenta tanto los logros de los objetivos previstos como los no previstos. Esta evaluación de resultados permitirá, por una parte, reflexionar sobre el proceso de Intervención en Medio Abierto y , por otra, ayudar a tomar decisiones de cara al presente y al futuro de aquel menor y por último realizar un informe final.

La devolución de estos resultados al menor, la familia, a los profesionales que han colaborado, así como al juez, comporta uno de los elementos más significativos que permite a las partes tomar conciencia de todos sus planteamientos anteriores y de las modificaciones que se tienen que hacer de cara a un futuro.

\section{Rol del menor}

\section{Autonomía e Integración social}

Ya indicamos que los objetivos que planifica el DAM no son objetivos finalistas, en el sentido de resolver toda la problemática del menor, sino más bien son obje- 
tivos que ayudan al menor a encontrar los puntos ċlaves en su problemática que contribuyan a clarificarla y a alcanzar aquellos objetivos específicos que le faciliten el proceso de integración social. En esta fase del proceso, los esfuerzos por parte del menor se centran en lograr una mayor integración social y una menor dependencia del DAM, ya que próximamente acabará su relación. Lo importante es que el menor comprenda: el proceso que se está llevando a cabo con él y la mejoría que ha experimentado, se haga conciente de las habilidades y objetivos que ha conseguido. En este sentido se trata que el menor vea que ha ido adquiriendo una mayor autonomía y que este cambio que ha realizado se lo ha de atribuir a sí mismo.

\section{Tipos de estrategias, técnicas y babilidades}

Podemos decir que las estrategias son similares a la fase anterior aunque pretendan distintos objetivos. Tal vez una de las estrategias más típicas de esta fase es el posible distanciamiento de los contactos. El DAM, para favorecer la autonomía del menor, realiza un tipo de seguimiento más indirecto a través de contactos con las personas que se relacionan con él. Los contactos con el menor, pueden estar más distanciados en el tiempo, y con ello puede facilitar una mayor autonomía y responsabilidad.

Estrategias, técnicas y habilidades de relación directa

El auto-refuerzo. La utilización de los refuerzos es una técnica utilizada en todas las fases, pero en esta fase final es importante conseguir que los menores aprendan a autoadministrarse sus propios refuerzos como una autoevaluación positiva por haber alcanzado unos objetivos concretos.

La inyección de perspectivas. Consiste en introducir nuevas perspectivas de futuro. Sugerir posibles alternativas que puede utilizar. Orientar sobre maneras prácticas y realistas de cómo se puede hacer frente a las situaciones presentes y a las futuras.

La entrevista sigue siendo la técnica que se utiliza fundamentalmente, tanto para la recogida de información que nos puede facilitar la evaluación sumativa, como para el establecimiento de la relación de ayuda.

La empatía. En esta fase la comprensión empática alcanza los niveles máximos de significación. El DAM puede utilizar la empatía añadiendo un significado más profundo en sus expresiones y ello facilita que el menor sea capaz de expresar y sentir sentimientos que fue incapaz de expresar anteriormente. Se supone que la comprensión empática en los niveles más elevados se convierte en acción constructiva para la resolución de problemas.

La aceptación. Esta última fase se caracteriza por un intento de que el menor logre una mayor autonomía y que pueda continuar, si es preciso, el proceso educativo en otras alternativas. En este sentido, el DAM utiliza la aceptación de una forma condicional. Es decir reforzando aquellos aspectos positivos que manifiesta el menor y no aceptando sus actitudes negativas.

La confrontación. En la última fase la confrontación toma todo su sentido. El menor es ayudado a través de las confrontaciones a enfrentarse, si es necesario, consigo mismo y con los otros. Aprende, a través de la confrontación de las discrepancias, a responsabilizarse de sus decisiones e implicaciones. Ayudando al menor a que aprenda cómo resolver sus problemas, independientemente del DAM, aumentaremos su libertad. Los menores pueden entonces asumir el control de sus propias vidas y hacerse más autosuficientes en la resolución de sus problemas futuros.

Estrategias, técnicas o habilidades de relación indirecta: Búsqueda o continuación de recursos educativos

El tiempo de intervención del DAM está limitado por la duración de la medida. Por lo general el menor ya está integrado en un recurso, pero el Delegado tiene 
que preveer, si el menor lo necesita, la continuidad en este recurso o la derivación a otro tipo de recursos que puedan seguir la labor de integración social del menor. Para ello necesita establecer contactos con los nuevos profesionales que se responsabilizarán. Necesita motivar a la familia para que inicie o continúe el apoyo al plan de trabajo y preparar al menor informándole de todas las posibilidades que tiene para continuar su proceso.

\section{Referencias}

AMORós, P. (coord.) (1993). Metodologia d'intervenció en Medi Obert. Centre d'Estudis Jurídics i Formació Especialitzada. Generalitat de Catalunya.

AMOROS, P. (coord.) (1994). Intervención en medio abierto. Orientaciones para su práctica. Barcelona: Fundació Jaume Callis.

BECK, A. et al. (1979). Cognitive Therapy for Depression. NuevaYork: Guilford.

DePARTAMENT DE JUSTf́CIA (1991). Projecte tèminic. Servei de Medi Obert. Generalitat de Catalunya.

GUASCH, M. (1991). La actuación educativa en el ámbito judicial. Un modelo socio-cognitivo de intervención en libertad. Tesis Doctoral inédita. Facultad de Filosofía y Ciencias de la Educación de Tarragona. Universidad de Barcelona.

MeinCHebBaum, D. (1977). Cognitive Bebavior Modification. Nueva York: Plenum Press.

RIMBaU, C. (1988). Els Dam, un projecte d'intervenció social amb perspectives de futur. Centre d'Estudis jurídics i Formació Especialitzada. Generalitat de Catalunya.

\section{El proceso de intervención en medio abierto: la libertad vigilada \\ Pere Amorós \\ CL\&E, 1995, 27, pp. 75-86}

Resumen: En este artículo se presenta el proceso de intervención que se puede urilizar para llevar a cabo la medida de Libertad Vigilada. Este proceso surgió de una investigación cooperativa llevada a cabo en Cataluña con todos los Delegados de Asistencia al Menor, que llevaban más de diez años ejecutando estas medidas judiciales para jóvenes infractores.

Datos sobre el autor: Pere Amorós Martí es Profesor Titular de Pedagogía de la Inadaptación Social.Facultad de Pedagogía. Departamento MIDE. Universidad de Barcelona.

Dirección: Departamento MIDE, Facultad de Pedagogía, Universidad de Barcelona, Baldiri Reixac, s/n. 08028 Barcelona. Tel. (93) 4409200.

(C) PERMISOS PARA CITAR O REPRODUCIR EN OTRAS FUENTES: Se pueden citar libremente hasta 500 palabras. Para reproducir una porción de texto mayor, figuras o ilustraciones, se deberá pedir permiso por escrito a la revista, especificando el uso al que se destina el texto. En todos los casos, se deberá citar el copyright de $C L \& E$. En el caso de artículos o textos que hayan sido a su vez reproducidos en $C L \& E$ los interesados deberán dirigirse tanto a los detentadores del copyright original como a $C L \& E$, en el caso de que se quiera hacer uso de la traducción. FOTOCOPIAS: Para todo lo relacionado con el uso mediante fotocopia del material de esta revista, deberán dirigirse a: CEDRO, C/ José Marañón, 10, $3 .^{\circ}$ Izda. Tel. 5941575 . Fax 4453567 